\title{
Avaliação da Usabilidade da Tecnologia Eye Tracking
}

\author{
Usability Assessment of Eye Tracking Technology
}

\author{
Letícia Simões Ferreira ${ }^{1}$, Leslie Andrews Portes ${ }^{2}$, Monalisa Pereira \\ Motta $^{3}$, Abrahão Augusto Joviniano Quadros ${ }^{4}$, Francis Meire Fávero ${ }^{5}$, \\ Acary Souza Bulle Oliveira ${ }^{6}$, Vagner Rogério dos Santos ${ }^{7}$
}

\begin{abstract}
1.Fisioterapeuta, Especialista em Neurologia Clínica e Doenças Neuromusculares, voluntária no ambulatório de Esclerose Lateral Amiotrófica do Setor de Doenças Neuromusculares da Unifesp. São Paulo-SP, Brasil.

2.Fisiologista do Exercício, voluntário do Departamento de Fisiologia Cardíaca da Unifesp. São Paulo-SP, Brasil.

3.Fisioterapeuta, Mestre, voluntária no ambulatório de Síndrome Pós-Poliomielite do Setor de Doenças Neuromusculares da Unifesp. São Paulo-SP, Brasil.

4.Fisioterapeuta Doutor em Saúde e Chefe do Ambulatório de Síndrome Pós-Poliomielite do Setor de Investigação em Doenças Neuromusculares da Unifesp. São Paulo-SP, Brasil.

5.Professora Afiliada do Departamento de Neurologia e Neurocirurgia da Unifesp. São Paulo-SP, Brasil.

6.Neurologista, Chefe do Setor de Investigação em Doenças Neuromusculares da Unifesp. São Paulo-SP, Brasil.

7.Docente Adjunto do Departamento de Oftalmologia e Ciências Visuais da Unifesp. São Paulo-SP, Brasil.
\end{abstract}

\section{Resumo}

Introdução. A Usabilidade se define como a facilidade do usuário em aplicar uma tecnologia a fim de realizar uma tarefa específica com eficácia, eficiência e satisfação. No desenvolvimento de Tecnologias Assistivas, é importante a inclusão do usuário, bem como a utilização de testes antes, durante a após a confecção destas visando obter altos padrões de usabilidade, evitando desgaste mental e psicológico e abandono de tecnologia. O objetivo foi avaliar a Usabilidade da Tecnologia Assistiva de Comunicação Aumentativa Eye Tracking. Método. Cinco profissionais da área da informática de ambos os sexos, com média de 5,7 anos de formação em: ciência da computação e análise e desenvolvimento de sistemas. Estes responderam um questionário para avaliação da Usabilidade após realizarem um teste com a tecnologia. Os testes foram divididos em etapas, com tempos determinados, abrangendo o recebimento, conexão dos itens, instalação, configuração dos softwares e realização de uma tarefa. Resultados. A maioria dos profissionais encontrou dificuldades no posicionamento e calibração do software e no idioma. Houve desistência de $20 \%$. Conclusão. Foi possível avaliar a usabilidade da tecnologia indicando caminhos para o aperfeiçoamento de um manejo mais eficaz, eficiente e satisfatório para o usuário.

Unitermos. Utilização; Tecnologia Assistiva; Comunicação não Verbal; Questionários; Avaliação de Desempenho; Satisfação do Usuário

\begin{abstract}
Introduction. Usability is defined as the ease of the user to apply a technology in order to accomplish a specific task with effectiveness, efficiency and satisfaction. In the development of Assistive Technologies, it is important to include the user, as well as the use of tests before, during and after making them aiming to achieve high usability standards, avoiding mental and psychological wear and abandonment of technology. The objective was to evaluate the Usability of Augmentative Communication Assistive Technology Eye Tracking. Method. Five computer professionals of both sexes, with an average of 5.7 years of training in: computer science and systems analysis and development. They answered a Usability assessment questionnaire after performing a technology test. The tests were divided into stages, with determined times, including receiving, connecting items, installing, configuring software and performing a task. Results. Most professionals found it difficult to position and calibrate the software and language. There was a $20 \%$ dropout. Conclusion. It was possible to evaluate the usability of the technology indicating ways to improve a more effective, efficient and satisfactory management for the user.
\end{abstract}

Keywords. Utilization; Assistive Technology; Non-Verbal Communication; Questionnaires; Performance Evaluation; User Satisfaction 
Trabalho realizado na Escola Paulista de Medicina, Universidade Federal de São Paulo - Unifesp, Campus São Paulo , São Paulo-SP, Brasil.

\section{INTRODUÇÃO}

A Usabilidade é considerada um conjunto de atributos de uma tecnologia, e está relacionada ao esforço necessário para seu uso e para a opinião individual de tal uso por determinado grupo de usuários. Estes atributos devem ser considerados mediante o tipo de usuário e as tarefas por ele executadas quando da interação com a tecnologia ${ }^{1}$.

Em outras palavras, a Usabilidade avalia a facilidade do usuário em aplicar uma tecnologia de informação ou ferramenta a fim de realizar uma tarefa específica. É um termo que também abrange os métodos de mensuração, bem como dos princípios envolvidos na eficiência de uma tecnologia. Pode ser aplicada até mesmo em ergonomia fazendo referência a design de objetos de uso cotidiano².

A Usabilidade deve ser alcançada com efetividade, eficiência e satisfação, constituindo as métricas de avaliação ${ }^{3}$. Os testes para avaliação de Usabilidade são realizados mediante as ferramentas e métricas específicas ${ }^{4}$.

A Usabilidade pode ser mensurada pela facilidade de aprendizado, onde o usuário de maneira rápida consegue explorar e desenvolver suas atividades; facilidade de memorização, onde o usuário é capaz de retornar a utilizar 
a tecnologia mesmo após certo tempo sem manter contato e sem necessidade de reaprender a manejar; e baixa taxa de erros, onde o usuário realiza suas tarefas sem desconfortos e consegue reparar erros se caso eles ocorram ${ }^{3}$.

Diante disto, estabeleceu-se alguns critérios para a avaliação da Usabilidade:

- Analisar as características da tecnologia em um contexto de uso específico;

- Analisar a interação entre usuário e tecnologia;

- Analisar a eficiência, a eficácia e a satisfação do uso da tecnologia².

O termo Acessibilidade, no tocante a tecnologias com softwares e aplicativos, refere-se a regras e recomendações que devem ser observadas durante o desenvolvimento de programas para computador ou aplicativos para celulares que possam ser utilizados por todas as pessoas que tenham deficiência ou não, evitando possíveis "barreiras" de comunicação. Com isso, um software é acessível quando possui funções que atendam usuários com perfis diferenciados ao passo que a Usabilidade avalia uma atividade funcional específica ${ }^{5}$.

Atualmente vários softwares já estão disponíveis no mercado, distribuídos gratuitamente e com muito boa aceitação por seus utilizadores. São programas que auxiliam na manipulação das funções básicas de sistemas operacionais, bem como os aplicativos que acompanham normalmente o sistema operacional, projetados de forma a 
atender as necessidades especificas, possibilitando a movimentação de dispositivos de inputs de dados como: mouses, teclados virtuais e sistemas de som².

Para saber se um software está pronto para o uso, é importante avaliar a interface. Quanto antes os problemas de interação ou interface forem descobertos e avaliados, menor o custo em corrigi-los ${ }^{6}$.

O profissional que desenvolve a tecnologia não pode presumir que os usuários sejam como ele, e que apenas a sua própria avaliação ou ideia seriam o suficiente para confirmar a qualidade de um produto final. Portanto, uma avaliação à parte é essencial para identificar as necessidades de um usuário, possíveis problemas de interação e as interferências da interface no momento do trabalho do usuário. Isso permitirá comparar outras alternativas do projeto, alcançar bons resultados em Usabilidade e verificar a conformidade com um modelo padrão ${ }^{2}$.

Como roteiro de avaliação, é proposto os seguintes passos:

- Determinar os objetivos gerais que a avaliação deverá tratar;

- Explorar perguntas específicas a serem respondidas;

- Escolher o paradigma e as técnicas de avaliação que responderão as perguntas;

- Identificar questões práticas que devem ser tratadas;

- Decidir como lidar com questões éticas;

- Avaliar, interpretar e apresentar dados ${ }^{7}$. 
O desempenho é um fator importante a ser avaliado, podendo estipular uma tarefa com um determinado tempo. Estas tarefas devem representar o sucesso do próprio usuário. Prevê-se que sejam realizadas comumente pelo usuário durante sua interação ${ }^{2}$.

Uma avaliação útil para tecnologias com softwares, consiste na avaliação analítica onde através da inspeção, são buscados problemas de uso visando recomendar consertos e melhoras para o projeto. Isto torna a equipe de avaliação capacitada para projetos de interface voltados a usuários ${ }^{2}$.

A importância de avaliação de uma tecnologia é evidenciada através de algumas realidades. É mais caro convencer um cliente a voltar a utilizar uma tecnologia que abandonou devido à má usabilidade do que atrair um cliente novo. O custo é reduzido quando os problemas identificados pelos pesquisadores são apresentados e solucionados no início do processo de desenvolvimento da tecnologia. Quanto melhor o uso, menor a recorrência de chamadas à central de suporte, que possui um alto custo operacional $^{8}$.

Visando que a comunicação é uma função básica para o usuário, aqueles que apresentam alterações nesta função podem utilizar técnicas e métodos denominados de Comunicação Aumentativa e Alternativa $(\mathrm{CAA})^{9}$, sendo esta a integração de símbolos, recursos, técnicas e estratégias no incentivo à comunicação ${ }^{10}$, socialização, educação e lazer de Pessoas com Deficiência (PCD). 
Com o avanço das Tecnologias de Informação e Comunicação (TIC), os sistemas de CAA estão cada vez mais digitais ${ }^{11}$ e se tratando de usuários com limitação de comunicação, estes apresentam características distintas de uso e de avaliações ergonômicas especificas, o que implica no sucesso ou fracasso na utilização destas ${ }^{12}$.

Um aspecto inerente às TIC's é o conceito de interface, que é também conhecida como Human Machine Interface (HMI), no Brasil traduzido como Interface Homem-Máquina (IHM), definindo a parte de um programa de computador ou aplicativo para tablets e/ou telefones celulares ou mesmo botões, com os quais o usuário interage com o objetivo de utilizar a tecnologia ${ }^{13}$.

A Tecnologia Assistiva (TA) surge como uma das formas de potencializar o desempenho nas Atividades de Vida Diária (AVD) de um usuário, facilitando a execução de determinadas tarefas e promovendo a superação de barreiras físicas e psicológicas ${ }^{14}$.

Dentre os recursos de TA, encontram-se brinquedos, roupas adaptadas, computadores, softwares e hardwares especiais, que contemplam questões de acessibilidade, dispositivos para adequação da postura sentada, recursos para mobilidade manual e elétrica, equipamentos de comunicação aumentativa, chaves e acionadores especiais, aparelhos de escuta assistida, auxílios visuais, materiais protéticos e milhares de outros itens confeccionados ou disponíveis comercialmente ${ }^{15}$. 
A tecnologia Eye Tracking (ET) refere-se a um conjunto de equipamentos que entre outras possibilidades, utiliza raios infravermelhos para avaliar a movimentação e posição do globo ocular, em relação a um plano de referência, que está sendo observado ${ }^{16,17}$.

A luz infravermelha é usada para evitar o desconforto do usuário com uma luz forte - que por sua vez batem na pupila e voltam ao dispositivo, permitindo calcular com precisão para onde o participante está a olhar e ainda medir os movimentos oculares ${ }^{16,17}$.

Um dado importante é que os $E T^{\prime} s$ precisam ser ajustados às particularidades dos movimentos oculares de cada pessoa, através de um processo designado por "calibração"12.

Durante este procedimento, o sistema grava o centro da pupila e a relação córnea-reflexo como se referindo a uma coordenada específica que é apresentada na tela do sistema computacional. A calibração que em muitos casos devem ser realizados periodicamente, podem ser mais ou menos efetivo, se o software de controle do ET tenha sido concebido com maior rigor em relação as necessidades ergonômicas dos usuários ${ }^{12}$.

Depois de identificado o centro da pupila e a localização do reflexo da córnea pelo software de processamento de imagem, por meio de cálculos trigonométricos define o "ponto de visualização" ou "pointof-regard", ou o ponto no espaço para onde a pessoa está 
olhando, revelando para onde a atenção visual está dirigida ${ }^{16,17}$.

A tecnologia ET contém um grande potencial de aplicação numa ampla variedade de disciplinas e áreas de estudo. Aliada a softwares específicos, possibilita a substituição de dispositivos de entradas de dados como mouse e teclado, utilizados para movimentação do cursor na área da tela para o acionamento de funcionalidades computacionais $^{16,17}$.

\section{MÉTODO}

\section{Amostra}

Este estudo é do tipo observacional com segmento transversal e foi desenvolvido no Setor de Investigações de Doenças Neuromusculares (SIDNM) da disciplina de Neurologia Clínica do Departamento de Neurologia/Neurocirurgia da Universidade Federal de São Paulo (UNIFESP) e no Laboratório de Fisiologia do Exercício (LAFEX) da Universidade Adventista de São Paulo (UNASP). Este estudo foi submetido e aprovado pelo Comitê de Ética em Pesquisa 1092211014 da UNIFESP/Hospital São Paulo.

Com base nos resultados obtidos por meio de um grupo piloto prévio composto por cinco profissionais de diferentes áreas, participaram deste estudo cinco profissionais da área de tecnologia da informática de ambos os sexos, com média de 5,7 anos de formação nos seguintes cursos: ciência da computação $(80 \%)$ e análise e 
desenvolvimento de sistemas (20\%), onde $80 \%$ atuam como analistas de sistemas e $20 \%$ como programadores.

Os critérios de inclusão selecionavam residentes do estado de São Paulo e graduados na área de tecnologia da informática. Foram excluídos os que já conheciam a tecnologia $E T$ bem como profissionais recém-formados.

\section{Procedimento}

O Quadro 1 apresenta as fases do teste, bem como suas etapas e tempos pré-determinados.

O usuário recebia do avaliador uma Folha de Instruções para o teste que se iniciava ao receber em mãos a tecnologia $E T$ IRISBOND ${ }^{\circledR}$, modelo BET 2.0. A gravação do teste era encerrada quando o usuário concluía todas as etapas, ou caso extrapolasse o tempo na etapa de calibração ou não concluísse todo o teste dentro de três horas.

Com base na Escala de Likert $^{18}$, o Questionário de Usabilidade consistia em dez domínios que abrangiam questões sobre embalagem, conteúdo interno, manual de instruções, instalação, uso, facilidade e satisfação.

Esta metodologia se baseou nas três métricas de avaliação de Usabilidade, que são eficiência, eficácia e satisfação e se limita em avaliar apenas os passos necessários até a configuração correta do aparelho e dos softwares para a boa utilização. 
Quadro 1. Divisão do teste de Usabilidade.

\begin{tabular}{|l|l|}
\hline Fase 1 & \\
\hline Realização das Etapas & $\begin{array}{l}\text { Tempo } \\
\text { (min) }\end{array}$ \\
\hline $\begin{array}{l}\text { A- Recebimento da Eye-Tracking, conexão do pen drive, cópia dos } \\
\text { dois softwares para a Área de Trabalho do computador }\end{array}$ & 5 \\
\hline B- Instalação do Software Primma & \\
\hline C- Instalação do Eye-Tracking & 15 \\
\hline $\begin{array}{l}\text { D- Posicionamento e calibração do Software Primma } \\
\text { E- Configuração do Software Primma e habilitar o comando dos } \\
\text { olhos }\end{array}$ & 5 \\
\hline F- Instalação do Software Smart ${ }^{2}$ & 30 \\
\hline $\begin{array}{l}\text { G- Realização da tarefa digitando a frase: “Eu (nome) consegui } \\
\text { realizar a tarefa”. }\end{array}$ & 20 \\
\hline Fase 2 & 30 \\
\hline A- Preenchimento do Questionário de Usabilidade & 15 \\
\hline B- Ficha do Avaliador & ------- \\
\hline
\end{tabular}

1.Software Primma, com funcionalidades de cursor do mouse. 2.Software Smart, de Comunicação Aumentativa. As etapas A e B da Fase 2 não possuíam tempo determinado para serem realizadas.

\section{RESULTADOS}

Com base nas respostas dos usuários no questionário de usabilidade, 60\% deles não sabiam o que era CAA nem mesmo conheciam a tecnologia ET e afirmaram ser pouco segura a embalagem da tecnologia (Figura 1).

A maioria dos usuários afirmou que a tecnologia não continha um manual de instruções impresso, porém todos fizeram uso do manual de instruções virtual na etapa de configurações. Na etapa de instalação e calibração apenas $60 \%$ fizeram uso e $80 \%$ na etapa do posicionamento (Figura 2). 
Figura 1. Perfil do voluntário e segurança da embalagem.

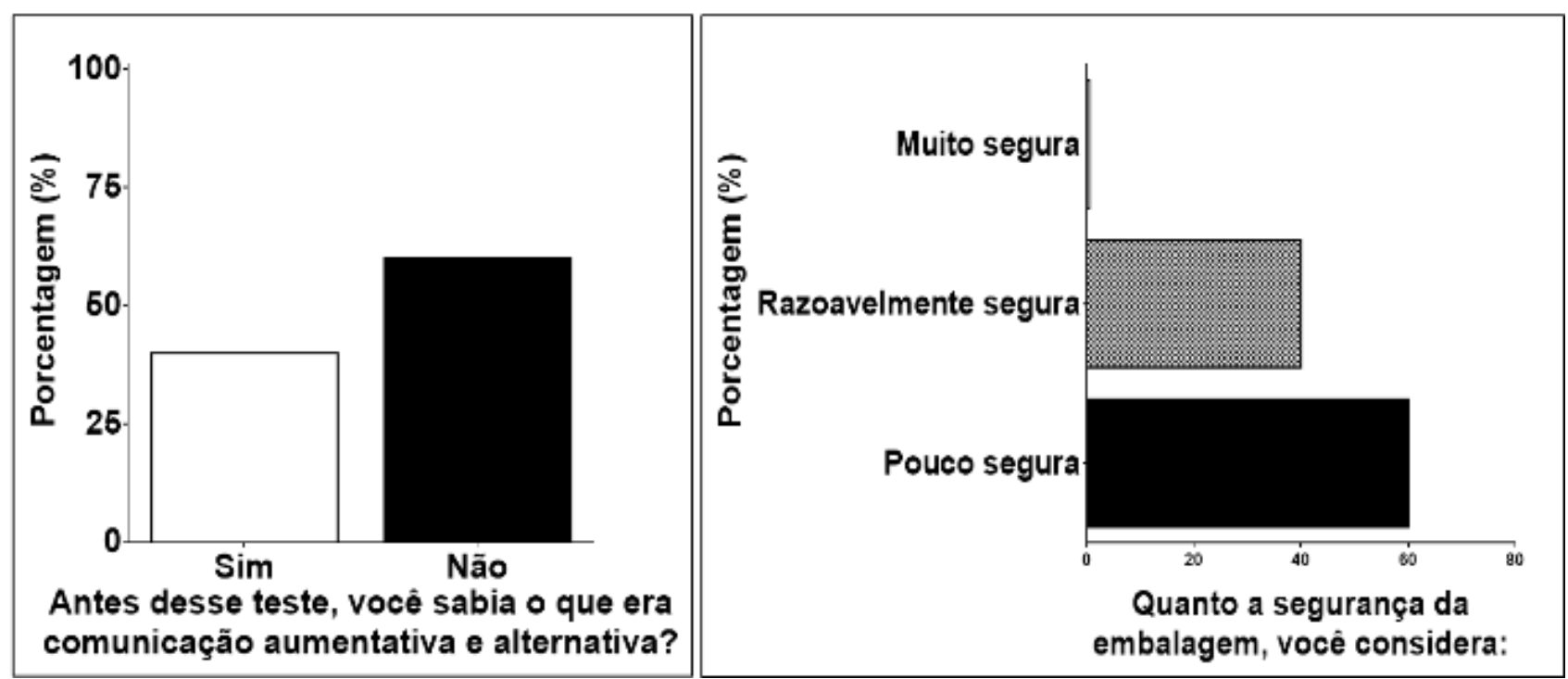

Figura 2. Fases de uso do manual de instruções.
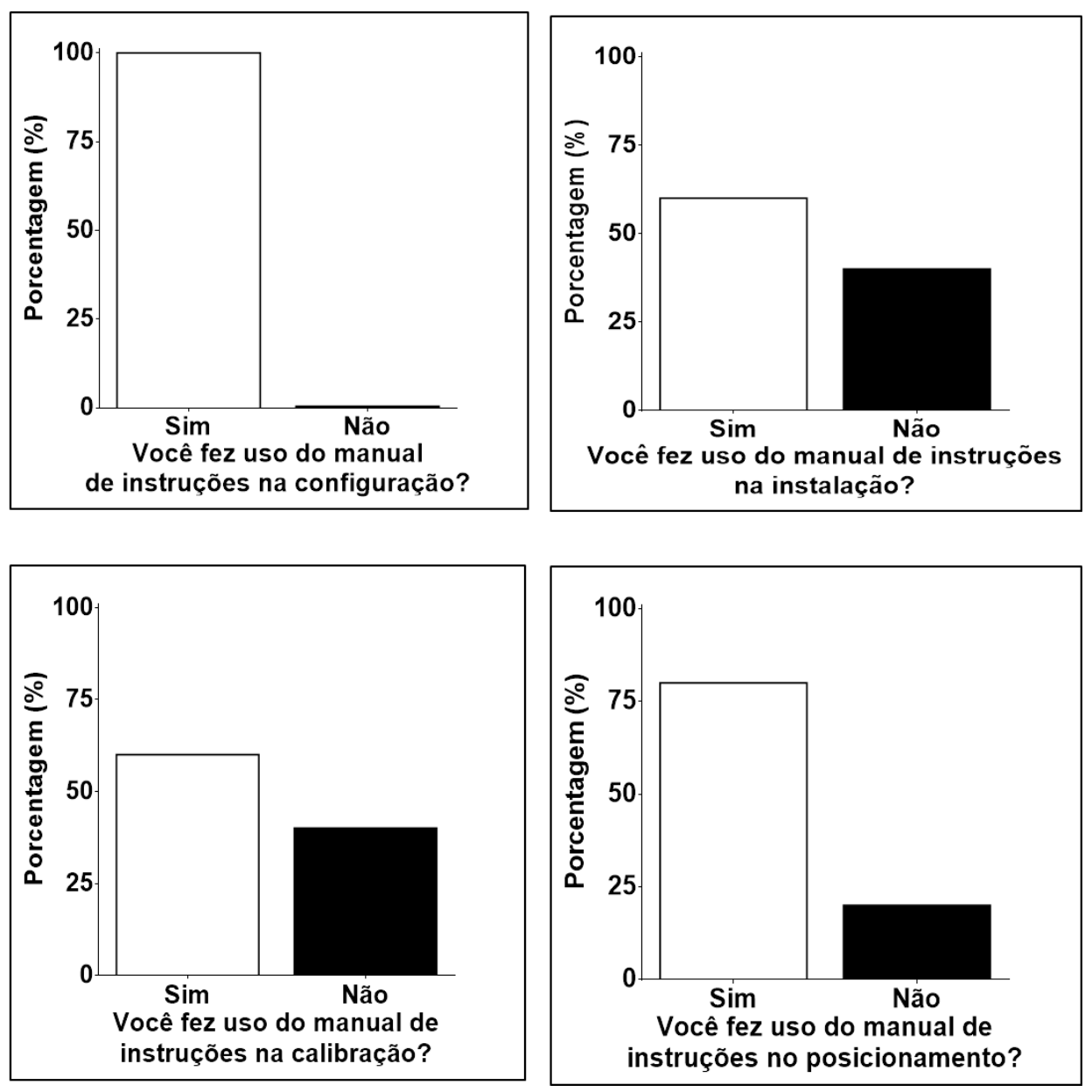
A maioria dos usuários (80\%) afirmou que o software Primma (Software com funções do mouse) funcionou corretamente contra $75 \%$ que afirmaram o mesmo sobre o software Smart (Software de CAA). Com relação a velocidade de carregamento dos softwares, 60\% avaliaram o software Primma como muito satisfatória contra 100\% muito do software Smart.

Vinte por cento dos usuários desistiram na etapa $\mathrm{F}$ e 20\% tiveram sua avaliação interrompida na etapa D. Quarenta por cento dos usuários selecionaram o modo "fixar" como clique; $100 \%$ fizeram uso do manual em alguma etapa, com predomínio na etapa D com $100 \%$ de uso. Nenhum participante reiniciou o computador ao final da instalação dos softwares conforme manual (Figura 3).

Figura 3. Modo de clique e uso geral do Manual de Instruções.
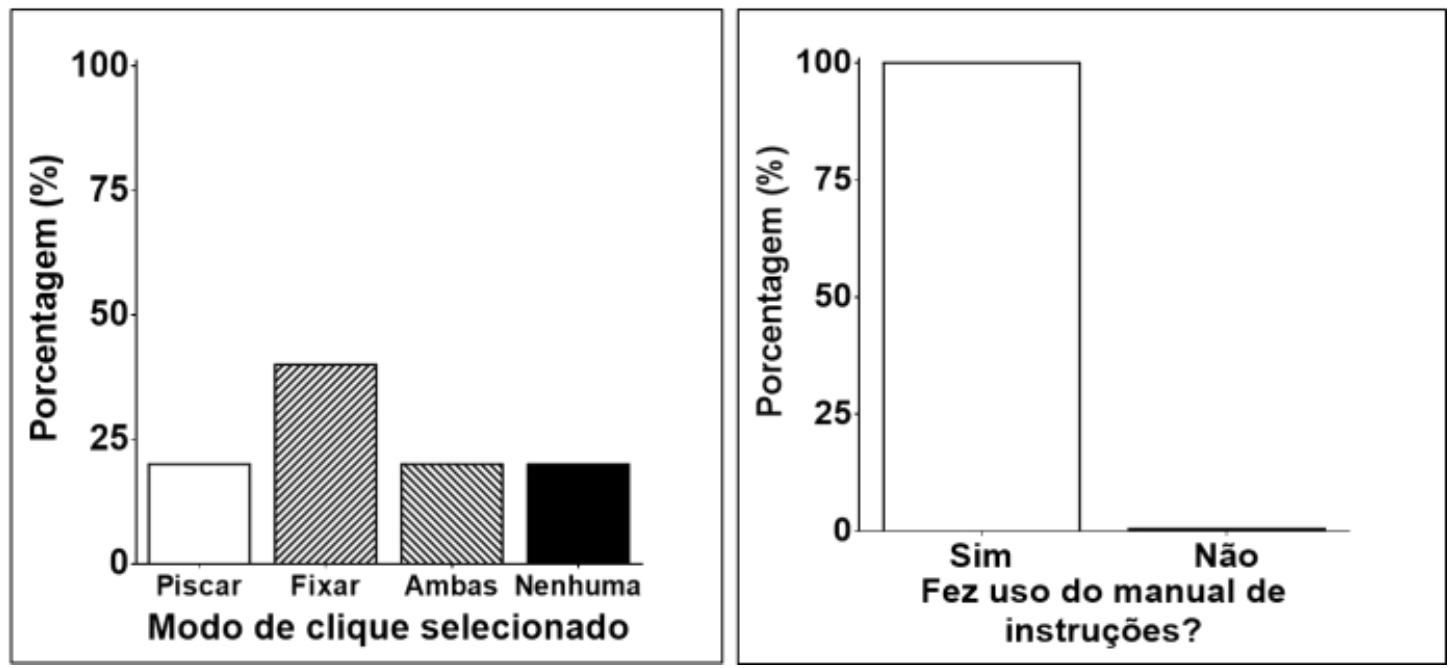


\section{DISCUSSÃO}

Algumas questões do Questionário geraram erros de interpretação e nenhum participante respondeu corretamente ao que uma pergunta específica solicitava. Nesta pergunta, os participantes deveriam enumerar as etapas em uma sequência de 1 a 7 onde " 1 " significava pouca dificuldade e "7" muita dificuldade, porém acharam que deveriam responder apenas com " 1 " e "7". Utilizandose um critério alternativo identificou-se que as etapas em que encontraram maior dificuldade foram a $D$ e $G$, com $80 \%$ das respostas.

Algumas respostas de outras questões não coincidiram com a realidade da embalagem do produto. Justificou-se este fato com a ideia de que alguns usuários não compreenderam que as perguntas eram relacionadas à embalagem externa do produto e não a embalagem dos itens internos em individual. Supõe-se também que alguns usuários não responderam com atenção devida, pois o questionário foi aplicado ao final do teste, que por sua vez era extenso e cansativo.

Sobre o manual de instruções, uma minoria considerou o tamanho das letras como não sendo ideal; o que poderia ser facilmente corrigido com o zoom do computador, uma vez que o manual era virtual. Quarenta por cento encontraram dificuldade no significado das palavras, "ecrã" e "mouse" presentes no manual. Julga-se que uma completa interpretação de todos os termos utilizados em um manual de instruções interfira 
diretamente na montagem de qualquer dispositivo, sendo um fator importante a ser levado em consideração no momento de elaboração do mesmo.

Existe uma preocupação em obter dados quantitativos para a medida da satisfação ${ }^{4}$. Neste estudo os domínios $\mathrm{H} \mathrm{e}$ I buscaram avaliar a satisfação dos usuários com relação aos softwares contidos no ET.

Pesquisadores que avaliam satisfação têm usado questionários, além da medição dos movimentos oculares que pode se mostrar útil na medição da satisfação, pois os olhos têm sido fortemente associados com as emoções, ou seja, a própria tecnologia ET pode ser usada como instrumento de medição de satisfação de algo em estudo, mediante o rastreamento ocular ${ }^{19}$.

O idioma foi alterado para o português pelo próprio avaliador em $60 \%$ dos casos, pois detectou-se a dificuldade dos usuários na etapa $\mathrm{F}$ com o comando dos olhos e com $\mathrm{o}$ tempo determinado para realizar a etapa.

Apenas um usuário encontrou problemas durante 0 teste, e por descuido excluiu todos os botões do cursor não conseguindo retornar à configuração-padrão para dar sequência. Diante do ocorrido, descobriu-se que não é possível desinstalar totalmente o software Primma de um computador. Foi necessário trocar de usuário no sistema, realizar outro login para instalar novamente o software e então prosseguir no teste. Esta descoberta justificou o fato de que em testes anteriores algumas fases da instalação não terem aparecido para o usuário, o que pode ter 
interferido diretamente no desempenho no teste, pois alguns se depararam com o idioma e as configurações já programados, facilitando a performance.

As etapas mais rápidas foram caracterizadas por atividades simples, tendo em mente que os usuários eram da área da tecnologia, que por sinal apresentavam muita habilidade e rapidez ao obedecer aos comandos.

As etapas A, B, C, D e G obtiveram uma média de tempo dentro da estipulada; sendo a etapa G a quarta etapa mais longa com apenas $25 \%$ dos usuários ultrapassando o tempo com $21 \mathrm{~min}$ e 40seg, o que mesmo assim não interferiu no extrapolamento do tempo médio calculado desta etapa. Em concordância com os resultados obtidos, as etapas F e D, com 30min e 55seg e 20min e $15 \mathrm{seg}$ de média respectivamente, foram as mais longas (Figura 4).

Figura 4. Tempo por etapa e etapa de maior dificuldade.
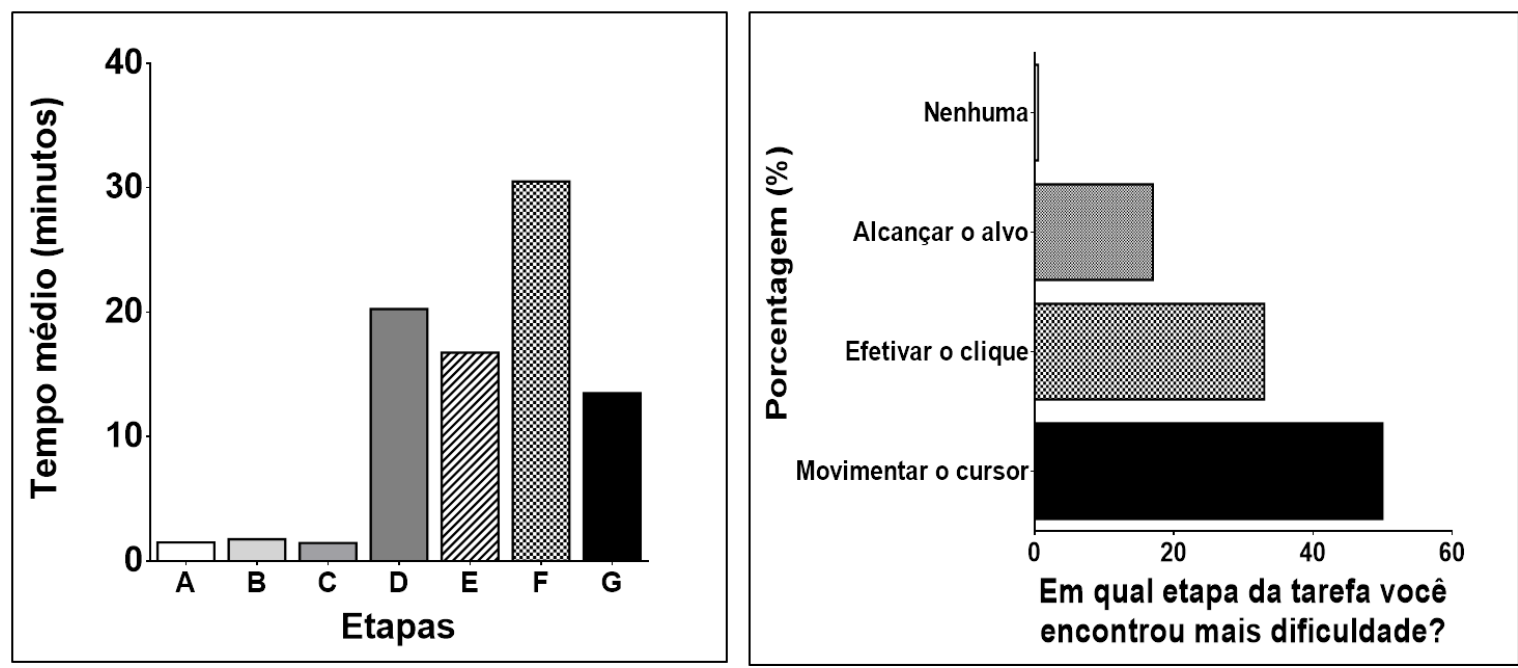
$\mathrm{Na}$ etapa $\mathrm{F}$ onde o comando dos olhos era primordial para instalação do software, os usuários não conseguiram realizar o que havia sido solicitado, portanto, o avaliador configurou os parâmetros necessários para a avaliação seguir de maneira mais confortável.

Isso pode ser justificado com a falta de leitura devida do manual para ajustarem corretamente os parâmetros necessários, ou pela fadiga do usuário. A frequência e duração das piscadas corresponde à performance da tarefa, e que mudanças nas métricas nas piscadas podem ser devidas à carga mental e fadiga do operador, induzidas pela tarefa ${ }^{20}$.

$\mathrm{Na}$ Figura 4 é possível comprovar que a etapa com maior dificuldade foi a $\mathrm{F}$ de calibração, com $60 \%$ dos casos.

Já a etapa E $16 \mathrm{~min}$ e $1 \mathrm{seg}$ com previsto de $15 \mathrm{~min}$, excedendo em um minuto do esperado devido um dos usuários que ultrapassou o tempo com 39min e 33seg, caracterizando a terceira etapa mais longa. O período de correção e de busca pela solução do problema trocando de usuário levou muitos minutos, tornando a etapa E deste usuário demasiadamente longa.

\section{CONCLUSÃO}

Conclui-se que este estudo foi capaz de avaliar e mensurar a Usabilidade da tecnologia $E T$, indicando caminhos para o aperfeiçoamento de um manuseio mais fácil e adequado para o usuário. 
Evidenciou-se que a tecnologia ET ainda não apresenta bons parâmetros de Usabilidade para auxiliar ao usuário no momento de instalação e configuração, mesmo tratando-se de usuários da área de tecnologia e experientes.

Existe a necessidade de realizar mais estudos sobre ajustes importantes com cada software para que a facilidade de instalação seja viável a todos os tipos de usuários. Além, da elaboração e adequação de ferramentas para análise de usabilidade voltadas para a área de CAA.

\section{AGRADECIMENTOS}

Programa de Apoio a Pós-Graduação e à Pesquisa Científica e Tecnológica em Tecnologia Assistiva no Brasil (PGPTA) - Edital No 59/2014.

\section{REFERÊNCIAS}

1.Dugalic B, Mishev A. ISO software quality standards and certification. CEUR Workshop Proc 2012;920:113-6.

2.Prates R, Barbosa $S$. Avaliação de Interfaces de Usuário-Conceitos e Métodos. Anais do XXIII Congr Nac da Soc Bras Comput [Internet]. 2003;1-4.

em:

http://homepages.dcc.ufmg.br/ rprates/ge_vis/cap6_vfinal.pdf

3.Standardization ISO. Ergonomics of human-system interaction. In: Part 11: Usability: Definitions and concepts. Online Browsing Platform; 2017.

4.Gobbi AG, Merino EAD, Merino GSAD, Gontijo LA. Uso do EyeTracking para obtenção de medidas quantitativas em testes de usabilidade. Um estudo focado na medida de satisfação. Hum Factors Des 2017;6:106-25.

http://dx.doi.org/10.5965/2316796306112017106

5.Ohira LM. Identificação de requisitos para usabilidade de software assistivo [dissertação]. Universidade do Paraná; 2009 p. 1-94.

6 .Karat $\mathrm{J}$. The cost-benefit and business case analysis of usability engineering. In: InterChi'93. Amsterdam: Tutorial NOtes 23; 1993.

7.Preece J, Rogers Y, Sharp E. Interaction Design: Beyond Human- 
computer interaction. New York: John Wiley and Sons; 2002.

8.MauroNewMedia. Professional Usability Testing and return on investiment as it applies to user interface design for web-based products and services (Endereço na Internet). Atualizado em: 2002; citado em 2016. Disponível em: http://www.taskz.com/ucd_testing_roi_summary.php

9. Mccoy KF, Waller A. Introduction to the Special Issue on AAC. J ACM Trans Access Comput 2009;1:13. http://dx.doi.org/10.1145/1497302.1497303

10.Santarosa LMC, Conforto D, Passerino LM, Estabel LB, Carneiro MLF, Geller M. Tecnologias Digitais Acessíveis. Porto Alegre: JSM Comunicações Ltda. 2010, 21p.

11.Ponte MN, Azevedo L. Comunicação Aumentativa e Tecnologias de Apoio. Edição CAPS/IST; 1998.

12.Goldberg JH, Wichansky AM. Eye Tracking in usability evaluation. A practitioner's guide. Elsevier Sci 2003;493-516. http://dx.doi.org/1016/B978-044451020-4/50027-X

13.Shneiderman $B$, Plaisant $C$. Designing the user interface: Strategies for Effective Human-Computer Interaction. 4th ed. Pearson Education India; 2010.

14.Bersch R. Recursos pedagógicos acessíveis. Tecnologia assistiva (TA) e processo de avaliação nas escolas. Assistiva; 2013.

15.Bersch R. Tecnologia assistiva. Porto Alegre: CORDE; 2013. p. 140.

16. Rele RS, Duchowski AT. Using Eye Tracking to evaluate alternative search results interfaces. In: Proceedings of the human factors and ergonomics society annual meeting. SAGE Publications 2005, p.145963.

17.Jacob RJK, Karn KS. Eye Tracking in Human-Computer Interaction and Usability Research: Ready to Deliver the Promises. Mind's Eye Cogn Appl Asp Eye Mov Res 2003;531-53. https://doi.org/10.1016/B978-044451020-4/50031-1

18. Likert R. A technique for the measurement of attitudes. Arch Psychol 1932;22:1-55.

19.Renshaw $T$, Stevens $R$, Denton PD. Towards understandingd engagement in games: An Eye Tracking study. Horiz 2009;17:40820. https://doi.org/10.1108/10748120910998425

20.Tsai Y, Virre E, Strychacz C, Chase B, Jung T. Task performance ans eye activity: predicting behavior relating to cognitive workload. Aviat Space Environ Med 2007;78(suppl 5):B176-85. 